\begin{tabular}{lcr}
\multicolumn{3}{c}{ ANNALES } \\
UNIVERSITATIS MARIAE CURIE-SKŁODOWSKA & \\
\multicolumn{2}{c}{ LUBLIN - POLONIA } \\
VOL. LXXI, 1 & SECTIO AA & 2016 \\
\hline
\end{tabular}

\title{
Modeling of adsorption phenomena by employing multilayer clustering based adsorption model (uniBET) with various variants of geometrical and energetical heterogeneities
}

\author{
Magda Ziółkowska ${ }^{\mathrm{a}^{*}}$, Janina Milewska-Duda ${ }^{\mathrm{a}}$ and Jan T. Duda ${ }^{\mathrm{b}}$ \\ AGH University of Science and Technology \\ ${ }^{a}$ Faculty of Energy and Fuels, Al. Mickiewicza 30, 30-059 Kraków, Poland \\ ${ }^{b}$ Faculty of Management, ul. Gramatyka 10, 30-251 Kraków, Poland \\ "email:magda.ziolkowska@agh.edu.pl
}

Multilayer clustering based adsorption model (uniBET) is aimed at adsorption phenomena modeling with having regard to surface geometrical and energetical heterogeneities. Facilitation of the uniBET model formulas leads to the so called LBET class models. A review on uniBET model and the analysis of the multilayer heterogeneous adsorption models for characterization of porous materials of carbonaceous origin are presented.

\section{INTRODUCTION}

Adsorbents of carbonaceous origin are widely applied porous materials for adsorption of gases and liquids due to its selectivity for many substances, high specific surface area and adsorption capacity [1]. These unique properties of adsorbents of carbonaceous origin and low cost production in comparison to the possibly competitive adsorbents of inorganic origin, results in a broad range of industrial applications. Thus, microporous carbonaceous materials are widely applied in gas separation, gas fuel storage processes, purification of water, as catalysis support or electrodes for fuel cells etc. 
The optimization of the industrial application of porous materials of carbonaceous origin is mainly related to the proper selection of the production process conditions [2-4]. Thus, precise, quick, low-cost examination techniques are required for the description (and prediction) of the microporous structure properties during the production process.

Most commonly experimental adsorption isotherms measurements are used for the characterization of adsorbent porous structure [1]. Nevertheless, in order to gain useful information on adsorbent parameters, the adsorption mechanism needs to be predetermined and described by the specific, corresponding mathematical model. There has been number of models already proposed for the adsorption equilibria description with different aspects of the adsorption mechanism being stressed. The most exploited technique is based on low pressure adsorption data employing BET equation that enable to evaluate material surface area $S_{\mathrm{BET}}$ [5]. BET theory is mainly criticized concerning an assumption of the energetical homogeneity of active adsorption sites, where adsorbate molecule may be placed and the independence of placing molecules in the neighboring adsorption sites (i.e. interaction between adsorbed molecules are neglected). Potential theories, dedicated mainly for microporous materials, namely Dubinin-Raduskievitch [6], Dubinin-Astakhov [7] enable to gain information on the pore volume and average surface energy. Nevertheless, for many adsorbents the fitting results of experimental data to e.g. DR model are unsatisfactory [8].

In order to avoid inadequacies of the application of BET and DR models, further development in adsorption modeling techniques is performed. In recent years, strongly recommended tool for the materials porous structure identification became density functional theory DFT [911]. Nevertheless, this model should be considered as a model dedicated mainly for the porous materials of regular structure. Thus, in case of highly irregular materials of carbonaceous origin (e.g. typical active carbons) fitting quality is comparable to the results obtained in our previous paper [12].

Moreover, the information on materials porous structure obtained from the application of the methods presented above is strongly affected by the type of the chosen probing adsorbate. Hence, our team elaborated new clustering-based multilayer adsorption theory (uniBET) comprising the surface geometrical and energetical heterogeneities to the analysis of the structure of microporous carbonaceous materials on the basis of experimental isotherms (gaseous and vapor). 
It was found out that the simplification of uniBET theory leads to the analytical formula of LBET class models, accurately describing adsorption system by fitting to the adsorption experimental data. The paper shows short review on uniBET theory, LBET class models formulas properties and its possible applications for microporous materials of carbonaceous origin.

\section{THEORETICAL BASIS AND uniBET FORMULA}

In clustering-based multilayer adsorption theory adsorption phenomena are viewed as a clusterization of adsorbate molecules in pores of different shape. The molecules of the first layer $n=1$ may be placed at the active adsorption sites on the pore wall, where adhesion energy reaches local minimum. The molecules of the further layers $n>1$ join with both adhesive and cohesive forces. For each $a$-th cluster involving $m_{p a}$ moles of adsorbate, adsorption equilibrium at a temperature $T$ and pressure $P$ may be described with the following formulae:

$$
R T \ln (\Pi)=\frac{\partial \Delta H}{\partial m_{p a}}-T \frac{\partial \Delta S}{\partial m_{p a}}
$$

where $R$ stands for the gas constant, $\Pi=f l f_{s}$ for the relative fugacity of the adsorbate in corresponding $P$ and $T$ and $\Delta H, \Delta S$ are total enthalpy and entropy change, respectively.

For the adsorption system satisfying an assumption of the configurationally invariant clusters creation, at the unique primary adsorption sites (that may be occupied only by one molecule), the total enthalpy change may be expressed:

$$
\Delta H=\sum_{a} m_{p a} Q_{a} \text { and } Q_{a}=\delta Q_{c}+U_{p}-Z_{a} Q_{c p}-Z_{p} Q_{p p}
$$

where $Q_{a}$ is an adsorption molar energy. The latter may be derived by adding the energy required to make room for the adsorbate particle $\delta Q_{c}$, cohesion energy for separation of an adsorbate molecule from the volatile phase $U_{p}$, adhesion energies for adsorbate-adsorbent $Q_{c p}$ and adsorbentadsorbent $Q_{p p}$ contacts, and the correction factors for energies resulting from geometrical conditions $Z_{a}$ and $Z_{p}$ (more detailed see ref. [13]).

The substitution of eq. (2) and formulae derived for $\Delta S$ in ref. [14] in the general formulae presented in eq. (1) leads to the set of algebraic 
equations constituting uniBET model, being a far generalization of the classical BET equation. The uniBET model describes the local adsorption isotherm on $\kappa$-th type primary sites, where $\kappa$ stands for the energy profile in cluster along the layers $n=1, \ldots, k$. Thus, the pair $\kappa, n$ refers to the $a$-th adsorption cluster and for the consecutive layers $n$ the uniBET model takes the form:

$$
-\Pi_{k n}^{*}+\left(\Pi_{k n}^{*}+1\right) \cdot \theta_{k n}-\theta_{k n} \cdot \theta_{k n+1}=0
$$

where $\theta_{\kappa n}$ is the coverage ratio of (n-1)-th layer in clusters of $\kappa$-th class:

$$
\theta_{k \imath} \stackrel{\text { def }}{=} \frac{m_{p k n}}{\beta_{k \imath} \cdot m_{p k n-1}}
$$

$\Pi_{k n}^{*}$ is a transformed pressure depending on the relative fugacity $\Pi, \theta_{\kappa n}$ stands for the coverage ratio and $\beta_{\kappa n}$ is pore compactness parameter:

$$
\Pi_{k n}^{*} \stackrel{\operatorname{def}}{=} \frac{\Pi}{B_{k n}}\left(1-\theta_{k l}\right)^{1-\beta_{k n+1}}
$$

$B_{\kappa n}$ denotes the energetic parameter of $\kappa n$-th subsystem:

$$
B_{k l} \stackrel{\text { def }}{=} \exp \left(Q_{k l} / R T\right)
$$

The parameter $\beta_{\kappa n}$ stands for the clusters branching factor and enable to consider not only stack-like clusters (as considered in the classical BET theory) but also possible branched clusters creation. This significant development is of essential influence on the configurational entropy of adsorption and may affect adsorption isotherms shape [14-15].

\section{LBET CLASS MODELS}

The uniBET theory makes possible to consider any distribution of the adsorption primary sites and energy profiles for each $\kappa n$-th type clusters. Nevertheless, in order to make practical use of the uniBET theory for examination of adsorption systems, it is necessary to reduce the number of fitting parameters. This leads to the class of adsorption models referred as LBET, comprising features of Langmuir and BET isotherms. 
However, in order to simplify the model, an additional assumptions need to be accepted for random microporous structure [13]:

- adsorption primary sites are exponentially distributed, where $m_{h A}$ is the total number of primary sites, expressed as a ratio of adsorbate volume $V_{h A}$ at the first layer and adsorbate volume $V_{s}$ at the adsorption system. $\alpha$ is an empirical distribution parameter:

$$
m_{h A k}=m_{h A}(1-\alpha) \alpha^{k-1} \text { and } m_{h A}=\frac{V_{h A}}{V_{s}}
$$

- for irregular porous materials parameter $\beta_{\kappa n}$ may be replaced by the overall clusters $\kappa n$-th type parameter $\beta$, being a geometrical average of $\beta_{k n}$ for all layers. We found out, that typically for compact pores $\beta \in(1,1.3)$ and for narrow pores $\beta \cong 1$.

- for $n=1$ adsorption energy at the first layer $\left(Q_{k l}=Q_{A}\right)$ is expressed:

$$
Q_{A}=U_{p}-Z_{A} Q_{c p}, \text { thus } B_{A} \stackrel{\text { def }}{=} \exp \left(Q_{A} / R T\right)
$$

where $\mathrm{Z}_{A}$ stands for the effective intermolecular contact at the first layer (and so, for a surface roughness) and $Q_{c p}$ is an adhesion energy as in eq. 2, calculated with the Berthelot rule [4].

- for $n>1$ a constant branching factor $\beta$ and the same adsorption energy $Q_{k n}=Q_{C}$ are taken:

$$
Q_{C}=U_{p}\left(1-2 Z_{p p}\right)-Z_{C} Q_{c p}, \text { thus } B_{C} \stackrel{\text { def }}{=} \exp \left(Q_{C} / R T\right)
$$

where $Z_{a a}$ is the correcting factor for adsorbate interaction contacts, $Z_{C}$ is an averaged correcting factor for adsorbate-adsorbent contacts at the layers $n>1$. Hence, it is assumed that layers $n>1$ are homogenous [4].

The remaining distribution parameters are defined as a set of variant factors. Energy distribution factor is defined by $h=0, \ldots, 9$. Binary variable $d=\{0,1\}$ is aimed at an individual determination of double molecules if $d=1$. An option $\eta=1$ from the binary variable $\eta=\{0,1\}$ gives a narrower adsorption energy distribution.

The abovementioned assumptions leads to the LBET class model for heterogeneous surface, considered as a subsets of uniformly distributed primary sites with different corresponding value of $Z_{A}$ parameter. This leads to the formulae [4]: 


$$
\begin{gathered}
\frac{m_{p}}{m_{h A}}=(1-\alpha)\left\{1+\frac{1}{\ln \left(B_{A} / B_{f 1}\right)} \ln \left(\frac{B_{A}+\Pi}{\left.\left.B_{f 1}+\Pi\right)\right\}}\right.\right. \\
\times\left\{\alpha(1-\alpha)\left(1+\beta \theta_{2}\right)\right. \\
\times\left\{1+\frac{1}{\ln \left(B_{A 2} / B_{f 2}\right)} \ln \left(\frac{B_{A 2}\left(1-\theta_{2}\right)^{\beta}+\Pi}{B_{f 1}\left(1-\theta_{2}\right)^{\beta}+\Pi}\right)\right\} \\
+\alpha^{d+1}\left[d+(\beta \theta)^{d}\left(1+\frac{\beta \theta}{1-\alpha \beta \theta}\right)\right] \\
\times\left\{1-\frac{1}{\ln \left(B_{A 2+d} / B_{f \theta}\right)} \ln \left(\frac{B_{A 2+d}(1-\theta)^{\beta}+\Pi}{B_{f \theta}(1-\theta)^{\beta}+\Pi}\right)\right\}
\end{gathered}
$$

where $\theta$ refers to the coverage ratio for layers $n>1$ and $B_{A k}, B_{f k}$ are energy parameters:

$$
B_{A k} \stackrel{\text { def }}{=} \exp \left(Q_{A k} / R T\right), B_{f k} \stackrel{\text { def }}{=} \exp \left(Q_{f k} / R T\right)
$$

In order to obtain energy distributions for $h>2$ simplified formulas were proposed to evaluate $B_{f k}=B_{f \theta}$ (for $h=1$ and 2 the model is directly applicable):

$$
B_{f \theta} \stackrel{\text { def }}{=} B_{A} \exp \left(Q_{c p} / R T\right)\left(Z_{f \theta}-Z_{f \infty o}\right)
$$

where $Z_{f \theta}$ is an averaged value of the upper limits $Z_{f k}$ of the energy distribution.

$Z_{f \theta}$ may be expressed by the following formula:

$$
\begin{aligned}
& Z_{f \theta}=\frac{\left(Z_{A 2+d}-Z_{f \infty}\right)}{1-\frac{\ln (1-\alpha)-(\beta \theta)^{1+\eta} \ln (1-\alpha \beta \theta)+(d-\eta) \alpha\left(1-(\beta \theta)^{2+\eta}\right)}{\alpha^{1+d-\eta} \frac{1-\beta \theta}{1-\alpha}\left[d+(\beta \theta)^{d}\left(1+\frac{\beta \theta}{1-\alpha \beta \theta}\right)\right]}} \\
& +Z_{f \infty}
\end{aligned}
$$

For the $B_{A k}=B_{A}$ the model in eq. 10 is applicable to adsorbents with homogenous surfaces.

Moreover two adsorption types are distinguished in LBET class models. First adsorption type refers to the clusters creation limited by the competitive growth of another cluster started in the separated adsorption 
primary site i.e. clusters are treated independently. Second adsorption type refers to the limitations of the cluster enlarging by the pore shape and size (more detail see ref. [16]).

Obtained LBET class models for heterogeneous surface involve five fitting parameters $\left\{\alpha, \beta, Q_{A}, Q_{C}, V_{h A}\right\}$. Heterogeneity variants $h_{i}$ with the options offered by the binary factors $d$ and $\eta$ creates a LBET model variant. During the identification of the model parameters, distribution variant is fitted to the experimental adsorption data. All of the available variants for the adsorption of the $1^{\text {st }}$ and $2^{\text {nd }}$ type were established to yield the distributions related to the real materials. The reliability of the identification is assessed by the identifiability factor $w_{i d}$.

\section{THE APPLICABILITY OF THE LBET CLASS MODELS}

LBET class models were found out to be a useful tool in characterization of wide range of modern carbonaceous materials for industrial applications [4], [17-21]. They enable to get an insight into adsorption nature, identify the volume of the first adsorbate layer and analyze diagrams of the adsorption energy distribution at the first layer.

An interesting application of the LBET class model was presented by Kwiatkowski et. al in ref. [19]. Authors performed an analysis on the carbon foams preparation methods from furfuryl alcohol. For the obtained carbon foam samples porous structure was analyzed applying 30-variant version of LBET class models. The theoretical isotherms were fitted to the experimental data using the method of fast multivariant identification procedure including non-linear optimization with limitations, in order to minimize the smallest square fitting error.

The selected results of the fast multivariant fitting procedure to the experimental adsorption isotherms for carbon foam samples PX1 and PX2, prepared by chemical vapor polymerization (CVP) in $120^{\circ} \mathrm{C}$ and $150^{\circ} \mathrm{C}$, performed by Kwiatkowski et. al [19] are presented in Fig. 1 and 2.

In short, carbon foams were produced over the melted oxalic acid $\left(\mathrm{H}_{2} \mathrm{C}_{2} \mathrm{O}_{4}\right)$, placed onto the porous glass located at the half of height of closed vessel. Subsequently, furfuryl alcohol (Aldrich, $10 \mathrm{ml}$ ) was added to the vessel and the reaction was carried out for around $30 \mathrm{~min}$. The chemical vapor polymerization was performed in $120^{\circ} \mathrm{C}$ for PX1 sample and in $150^{\circ} \mathrm{C}$ for PX2. Then, the produced powder of both samples was carbonized at $800^{\circ} \mathrm{C}$ in the atmosphere of Ar. The examination of 
microporous structure was additionally supplemented by the SEM analyses [19].

The empirical isotherm: $\mathrm{N}_{2}$ - PX1; Type 2, $d=0, \eta=0, \mathrm{~h}=5, \alpha=0.37, \beta=3.00$
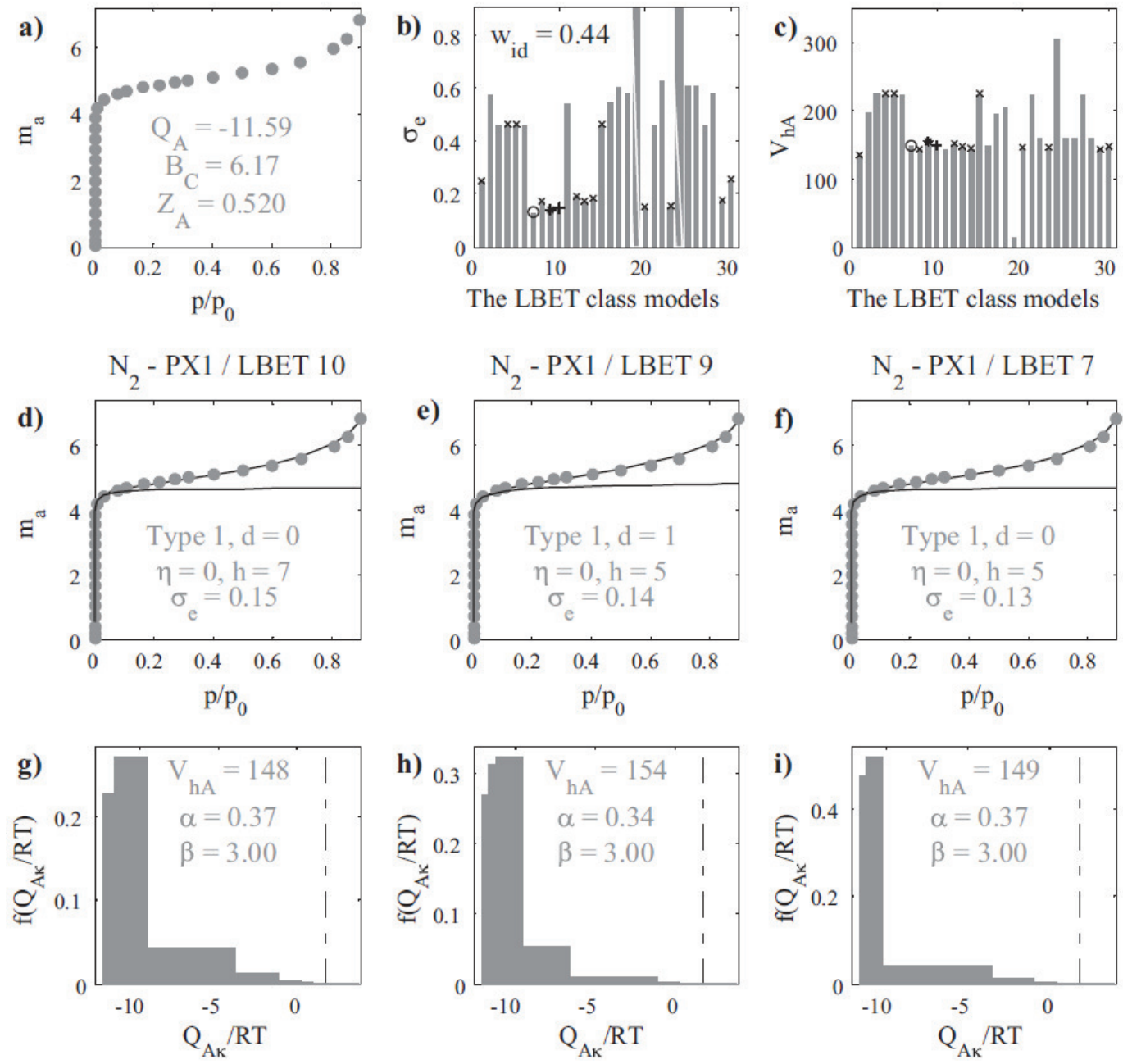

Fig. 1. The results obtained by Kwiatkowski et. al [19] for the empirical adsorption isotherm N2-PX1 (77 K).

In Fig. 1-4, at the (a) subfigure fitted empirical isotherm is presented, (b) is obtained error dispersion and (c) presents obtained values of the monolayer capacity $V_{h A}$, both for the calculated LBET class models variants. In figures (b) and (c) bold dot correspond to the optimal variant, " + " and "*" stand for the second and the third best fittings, respectively. In figures (d)-(f) three best fittings of the LBET class models are presented and in (g)-(i) the first layer adsorption energy distributions corresponding to the (d)-(f), respectively. Experimental data are denoted with the filled dots and fitted theoretical isotherm by the solid line. 
The empirical isotherm: $\mathrm{N}_{2}$ - PX2; Type 2, $\mathrm{d}=0, \eta=0, \mathrm{~h}=5 \alpha=0.26, \beta=1.05$
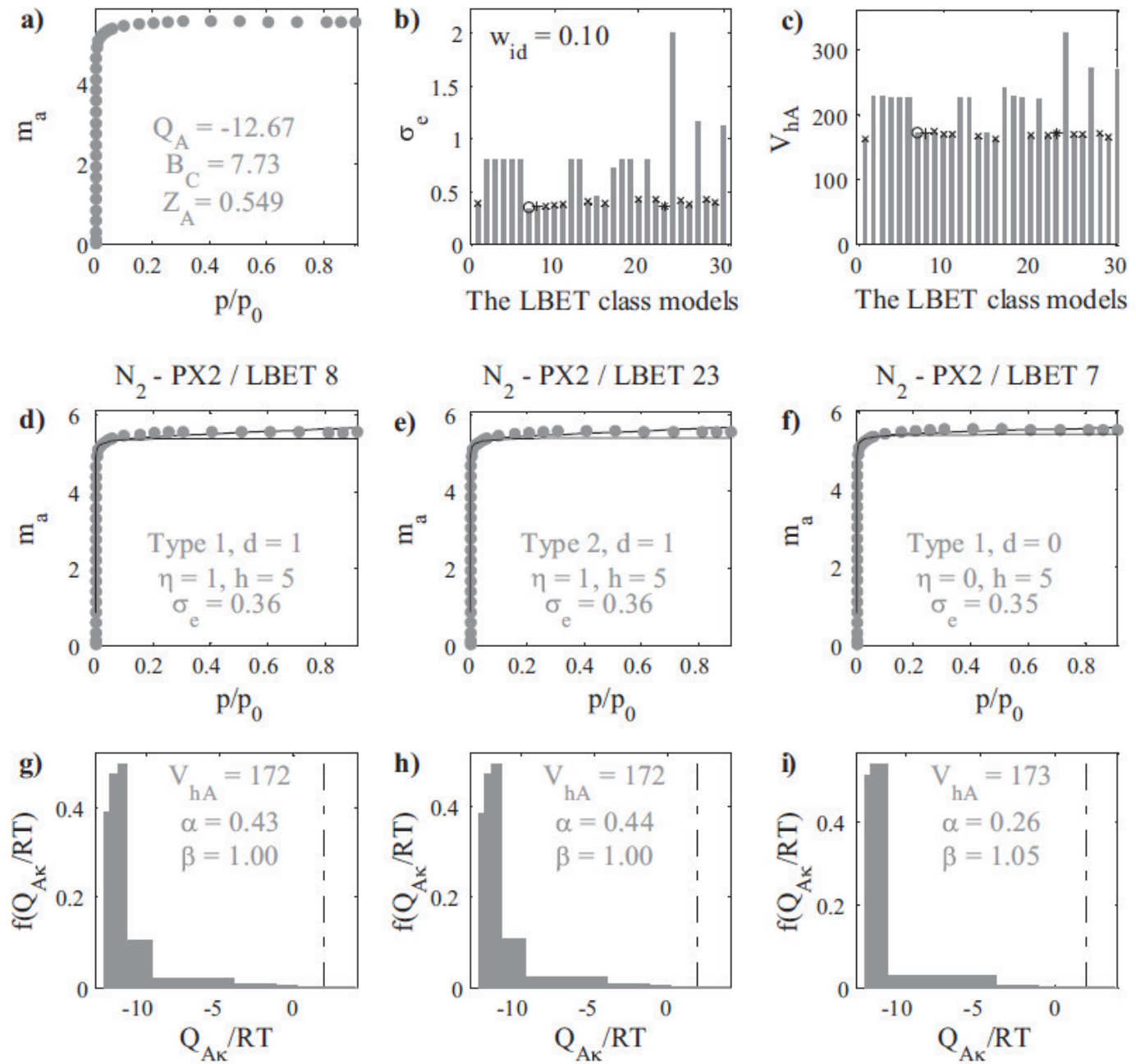

Fig. 2. The results obtained by Kwiatkowski et. al [19] for the empirical adsorption isotherm N2-PX2 (77 K).

Performed analysis for PX1 and PX2 provides useful information on the materials porous structure. For both samples it may be seen that analyzed material is strongly heterogeneous (high value of parameter $h=5$ ) with the main fraction of micropores - LBET 7 - best fitted variant.

Nevertheless, it was identified that preparation technique (i.e. in this case temperature) significantly affects porous structure. In PX1 sample low and branched clusters are predominant, due to high value of geometrical parameter $\beta$, whereas in PX2 narrow pores of stack-like shape were identified, i.e. significantly lower value of $\beta$ is observed. As evidenced by the type of the best fitted model, identified adsorption type is $1^{\text {st }}$. Thus, adsorbate clusters enlarging is limited by the competitive growth of the neighboring clusters. 
The attention should also be paid to the fitting reliability for both samples PX1 and PX2. For ten best fitted variants of LBET class models obtained values of first adsorption layer $V_{h A}$ are almost identical as it may be seen in Fig. 1. and 2. c). This feature is also confirmed by the obtained identical values of $\alpha$ and $\beta$ parameters for the three selected best fitted variants in Fig. 1. and 2. d)-f).

The empirical isotherm: $\mathrm{C}_{6} \mathrm{H}_{6}-\mathrm{CMS}$ (for the $\mathrm{KOH} /$ char treatment ratio (w/w) equal to 0:1)

a

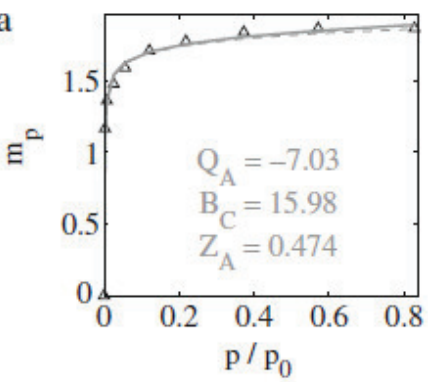

d
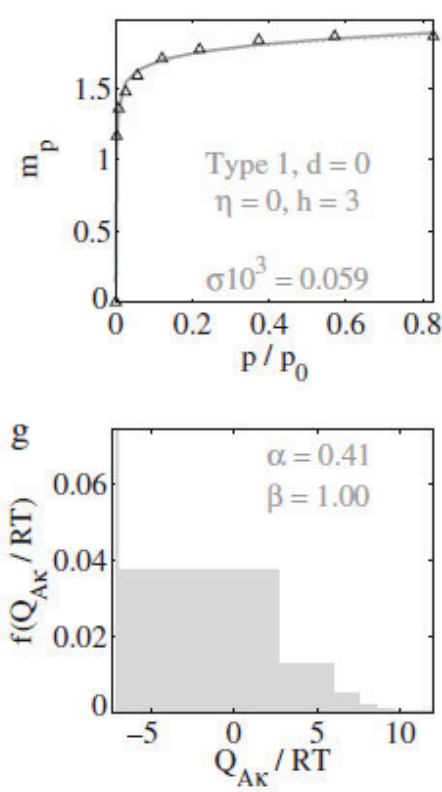

b

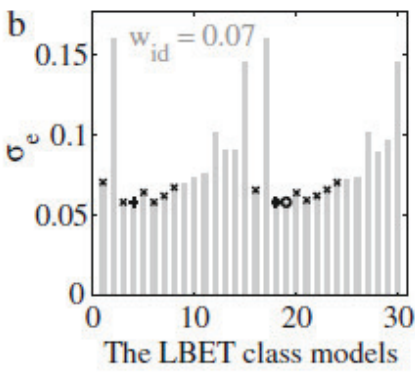

e
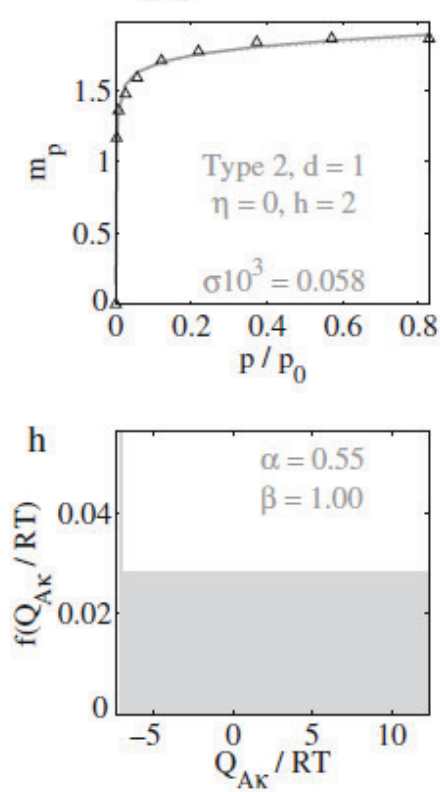

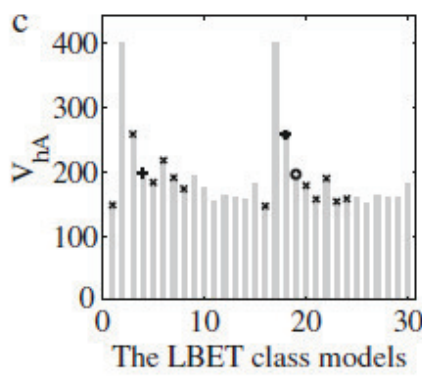

f $\quad \mathrm{C}_{6} \mathrm{H}_{6}-\mathrm{CMS} / \mathrm{LBET} 19$
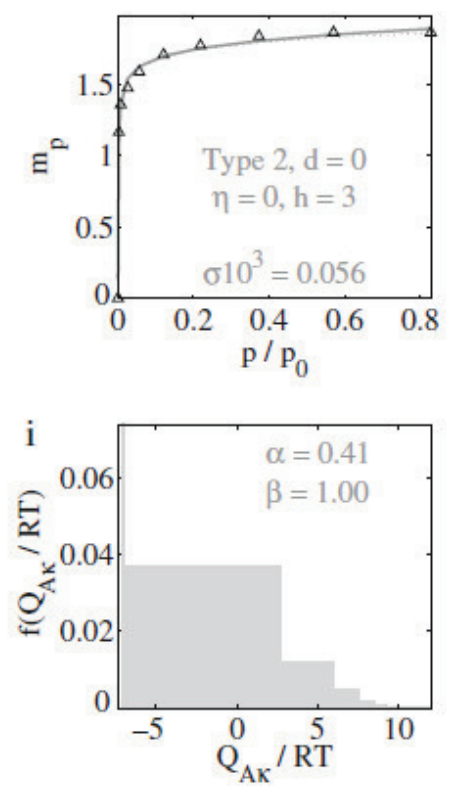

Fig. 3. The results obtained by Kwiatkowski [21] for the empirical adsorption isotherm $\mathrm{C}_{6} \mathrm{H}_{6}$-CMS (for the $\mathrm{KOH} /$ char ratio (w/w) 0:1).

Kwiatkowski [21] performed also an interesting analysis of the impact of activating agent on microporous structure formation in carbon molecular sieves (CMS) obtained from walnut shell. Numerical analysis was performed employing LBET class model with the procedure described above. The selected results of the fast multivariant fitting 
procedure to the experimental adsorption isotherms for CMS samples with $\mathrm{KOH} /$ charcoal ratio varied by weight $0: 1$ and 1:1 are presented in Fig. 3 and 4.

The empirical isotherm: $\mathrm{C}_{6} \mathrm{H}_{6}-\mathrm{CMS}$ (for the $\mathrm{KOH} /$ char treatment ratio (w/w) equal to 1:1)
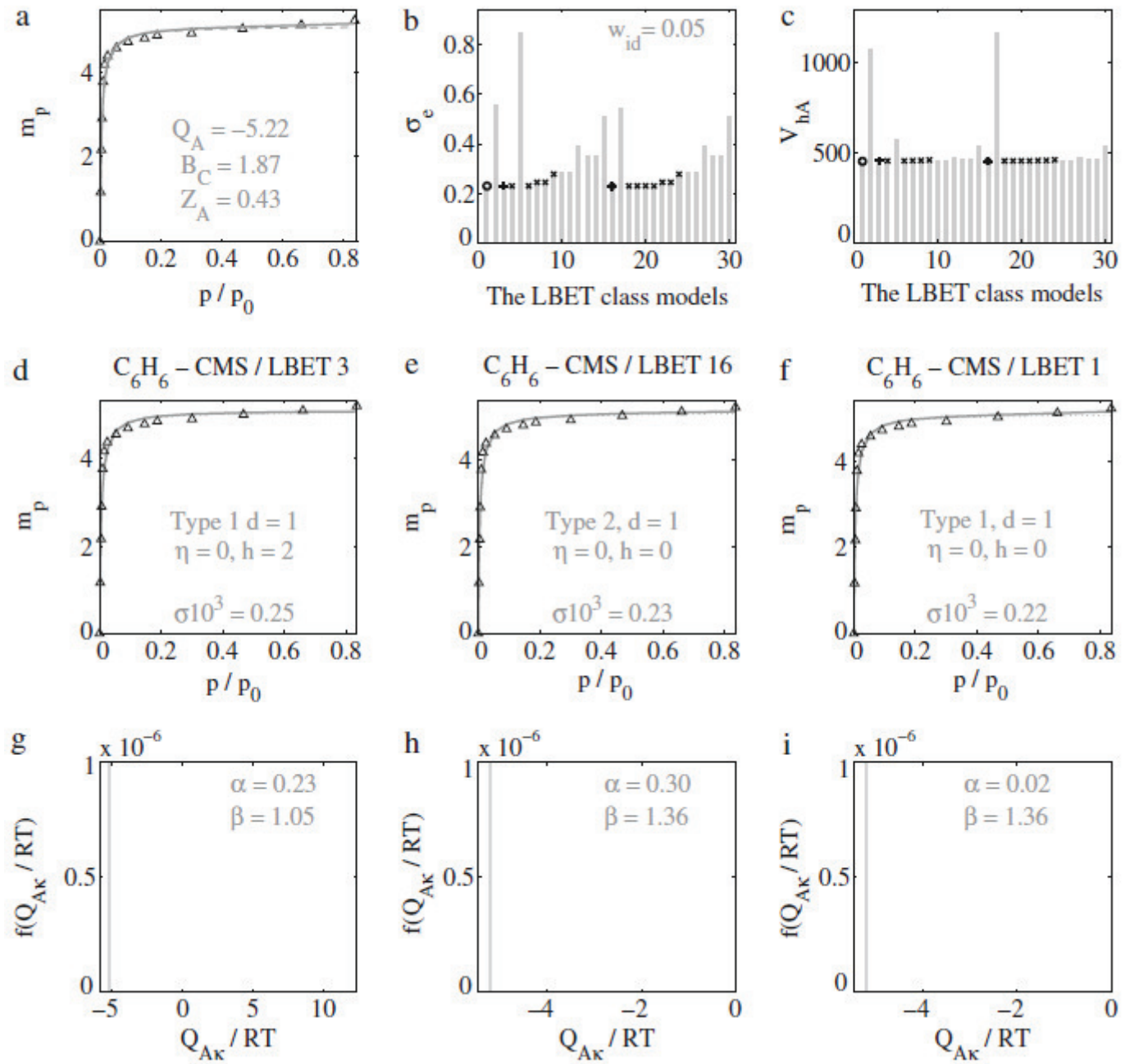

Fig. 4. The results obtained by Kwiatkowski [21] for the empirical adsorption isotherm $\mathrm{C}_{6} \mathrm{H}_{6}-\mathrm{CMS}$ (for the $\mathrm{KOH} /$ char ratio (w/w) 1:1).

The results of the performed analysis confirm adsorbents microporous structure and the influence of activating agent on the structure parameters. It may be qualitatively seen that monolayer capacity of the sample without the treatment of $\mathrm{KOH}$, presented in Fig. 3 is significantly lower than in case of the treatment with ratio by weight 1:1.

The treatment with an activator influences also surface heterogeneity, the significant heterogeneity in Fig. 3, i.e. $h=3$ may be observed, apart to 
the homogeneity increase noticeable in case of the sample treated with an activator. This feature may be also seen in Fig. 4. g) - i), where first adsorption layer energy is uniformly distributed, apart to the heterogeneous fractions observed in Fig. 3. g) - i). The values of geometrical parameters imply growth of the clusters, while activating agent is used. Thus, it enables to identify conical-like micropores.

The attention should also be paid to the obtained monolayer capacity $V_{h A}$, where almost identical values were produced for all LBET models variants, Fig. 4. c). Hence, this feature confirms again a homogeneity increase. Furthermore, activating agent treatment results in branched clusters development, where $\beta=1.36$ was identified.

\section{CONCLUSIONS}

The uniBET theory and LBET class models may be treated as a formal tool for the analysis of materials porous structure, assuming that the pore size may be accepted as an exponentially distributed and the monolayer capacity is closely related to the pore geometry.

Simplified LBET class models seems to be enough accurate to apply for the analysis of materials porous structure of real adsorbents. Moreover, multivariant fitting procedure is an effective tool enabling to avoid numerical problems coming from the large number of parameters.

In order to cover more exceptional cases of performed analysis the author's team leads researches on LBET class models extension. Also these extended versions were applied by Kwiatkowski and co-workers for porous materials structure evaluation. The numerical analysis of the carbon adsorbents structure, obtained from the ion-exchange resins, were also performed [20], as well as analysis of the production process conditions on microporous structure of active carbons [22]. Hence, LBET class models proves to be really useful tool in porous material characterization and enable also a qualitative and quantitative low cost examination of the material structure parameters and its comparison at the different stages of the production processes.

Hence, presented method will hopefully prove to be increasingly valuable for the technologists. 


\section{ACKNOWLEDGMENTS}

The research is led within the AGH University of Science and Technology grant No. 11.11.210.213.

\section{REFERENCES}

[1] A. Dąbrowski, Adv. Colloid Interface Sci., 93, 135-224, (2001).

[2] M. Bastos-Neto, D. V. Canabrava, A. E. B. Torres, E. RodriguezCastellón, A. Jiménez-López, D. C. S. Azevedo and C. L. Cavalcante, Appl. Surf. Sci., 253(13), 5721-5725, (2007).

[3] M. J. Prauchner and F. Rodríguez-Reinoso, Microporous Mesoporous Mater., 109(1-3),581-584, (2008).

[4] M. Kwiatkowski, J. T. Duda and J. Milewska-Duda, Colloids Surfaces A Physicochem. Eng. Asp., 457, 449-454, (2014).

[5] X. Lu, M. Jaroniec and R. Madey, Langmuir, 7(23), 173-177, (1991).

[6] M. M. Dubinin, Pure Appl. Chem., 61(11), 1841-1843, (1989).

[7] M. M. Dubinin and V. A. Astakhov, Bull. Acad. Sci. USSR Div. Chem. Sci., 20(1), 17-22, (1971).

[8] H. F. Stoeckli and J. P. Houriet, Carbon N. Y., 14(5), 253-256, (1976).

[9] J. Jagiełło and J. P. Olivier, Carbon N. Y., 55(2), 70-80, (2013).

[10] C. Lastoskie, K. E. Gubbins and N. Quirke, Langmuir, 9(3), 2693-2702, (1993).

[11] C. Lastoskie and K. E. Gubbins, Adv. Chem. Eng., 28, 203-251, (2001).

[12] J. T. Duda, L. Jagiełło, J. Jagiełło and J. Milewska-Duda, Appl. Surf. Sci., 253, 5616-5621, (2007).

[13] J. T. Duda and J. Milewska-Duda, Langmuir, 21(12), 7243-7256, (2005).

[14] J. Milewska-Duda and J. T. Duda, Langmuir, 17(15), 4548-4555, (2001).

[15] J. Milewska-Duda and J. T. Duda, Colloids Surfaces A Physicochem. Eng. Asp., 208, 71-81, (2002).

[16] J. T. Duda, J. Milewska-Duda and M. Kwiatkowski, Appl. Surf. Sci., 252, 570-581, (2005).

[17] M. Kwiatkowski, Langmuir, 23(16), 2569-2581, (2007).

[18] J. T. Duda, M. Kwiatkowski and J. Milewska-Duda, Appl. Surf. Sci., 256, 5243-5248, (2010). 
[19] M. Kwiatkowski, M. Wiśniewski and A. Pacholczyk, Colloids Surfaces A Physicochem. Eng. Asp., 385(1-3), 72-84, (2011).

[20] M. Kwiatkowski, M. Wiśniewski and G. Rychlicki, Appl. Surf. Sci., 259, 13-20, (2012).

[21] M. Kwiatkowski, J. Colloid Interface Sci., 340(1), 1-7, (2009).

[22] M. Kwiatkowski and E. Broniek, Colloids Surfaces A Physicochem. Eng. Asp., 411, 105-110, (2012). 